Website: ijetms.in Issue:5, Volume No.4, September-2020 DOI: 10.46647/ijetms.2020.v04i05.012

\title{
Comparison of Image Segmentation Methods in an Experimental Way
}

\author{
Ms.R.Vaishnavi ${ }^{1}$, Ms.S.Manjula ${ }^{2}$, Dr.K.Lakshmi ${ }^{3}$ \\ ${ }^{1}$ Final Year M.Sc (Software Engineering), \\ ${ }^{2}$ Assistant Professor, Department of Software Engineering \\ ${ }^{3}$ Professor, Department of Computer Science and Engineering, \\ 1,2,3 Periyar Maniammai Institute of Science and Technology (Deemed to be University) Thanjavur, Tamilnadu.

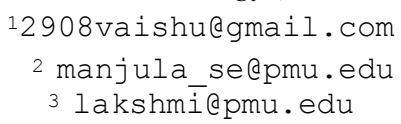

Abstract - Segmentation is an important procedure in image processing. It splits a digital image into numerous sections to examine them. It is also used to make a distinction in an image. Several image segmentation techniques are available to make a smoothen image and to examine easily. The main purpose of this work is to categorize the results and compares the threshold-based, edge-based, and watershed-based image segmentation methods. This work is executed by MatlabR2016a.

Keywords - clustering-based, Edge-based, Region-based, Threshold-based segmentation, and Watershed based segmentation.

\section{INTRODUCTION}

In image processing, the people interested in a certain portion of an image, commonly called Region of Interest. Image segmentation intends to learn the regions of preferred characteristics and region of interest in an image [1]. It is fundamental for further image processing works like image visualization, image analysis, and object detection, and so on. Many segmentation methods are available [2] - [4] and used on as per the choice of the problem domain. Many segmentation methods are available [2] - [4] and used on as per the choice of problem domain. The Edge-based segmentation, Threshold-based segmentation, Clustering-based segmentation, and Regionbased segmentation are widely used to extract the desired portions in an image.

The idea behind in segmentation is representing the similarity of pixels in any region of an image and discontinuity of edges in an image. The result of the image segmentation process is the main input parameter for further image processing tasks; this result determines the quality of further image processing process.

An image segmentation algorithm plays an important role in medical applications also. Hence, Image segmentation is still a very hot research area of the image processing field. It is still a challenging task for researchers and developers to develop a universal technique for image segmentation. This paper compares the edge, threshold, watershed segmentation methodologies of image processing.

\section{LITERATURE REVIEW}

\section{A. Segmentation Methods}

The clustering approach [3] [4] [5] is a process of grouping pixels with similar kinds of color, texture, and so on. The clustering approach was developed in [6] based on the assumption that the background would not be the part and appear in the sequence for a short duration. First, pixel intensities are classified based on an on-line clustering model. Then, the appearance probabilities of each cluster center are calculated. Finally, a single or multi intensities clusters with the appearance probability greater than a threshold are selected as the background image pixel. The algorithm is saving the first value of the cluster.Then, the current cluster value is compared to its previous value at every point of update to find out the deviation. If the deviation is bigger than a threshold, a new cluster is created from the past one and takes the weight of the current one. To optimize memory usage, the old clusters with no update are deleted based on the assumption that a background cluster is updated many times.

The basic clustering algorithms are divisive and agglomerative. It uses more number of pixels in an image and do not give the optimized solution. To solve this, the iterative $\mathrm{k}$-means clustering is used to minimize the variance between the pixel and cluster center. Hence, the optimal solution is based on the set of clusters and the value of $\mathrm{k}$. K-means models: Group of cluster is assigned to a pixel in an image, and it modifies by background and lighting variations. All the receiving pixels are mapped with the cluster group and are classified as whether the cluster is part of background or not. Genetic K-means algorithm is introduced by Duan et. al [7] reduce the lack of global optimization problem caused by traditional Kmeans algorithm.

Thresholding methods [2] [4] [8] [9] are simple and useful when the solid objects are present in a contrast background. It exactly defines the disjoint locations with connected closed boundaries. Based on the intensity values the image pixels are separated and it segregates the foreground, background and boundary locations. If the 


\section{International Journal of Engineering Technology and Management Sciences[IJETMIS]}

Website: ijetms.in Issue:5, Volume No.4, September-2020 DOI: 10.46647/ijetms.2020.v04i05.012

gray value in background is contrast throughout the image, global threshold works fine else local threshold gives good results.

Global threshold using an appropriate threshold T:

$$
g(x, y)= \begin{cases}1, & \text { if } f(x, y)>\mathrm{T} \\ 0, & \text { if } f(x, y) \leq \mathrm{T}\end{cases}
$$

If $g(x, y)=1$ is a foreground, otherwise it is a background. The global thresholding algorithms are 1.Otsu, 2.Optimal Thresholding, 3.Histogram Analysis, 4.Iterative Thresholding 5.Maximum Correlation Thresholding, 6.Clustering, 7.Multispectral and Multi thresholding. The variable thresholding is suitable where the background gray level is not uniform and contrast of object is varies. Otsu's threshold is iterates all possible threshold values. The variance is minimized within the class and maximized between the classes. Total variance $=$ within class variance + between class variance.

Edge detection methods [2] [3] point out the pixels in the image related to the edges of the objects in the image. An edge (image/object boundaries) is an important feature of an image it is known by a sudden change of discontinuities in intensity range. It distinguishes and separates images/objects from one another. It increases the sharpness of an image. Edge detection uses two detection operators. Gradient-based operator which computes first-order derivates of a digital image, those are 1.Sobel operator, 2.Prewitt operator, 3.Robert operator. Gaussian-based operator which computes second-order derivations in a digital image they are 1.Canny edge detector, 2.Laplacian of Gaussian. These algorithms are suitable for images that are simple and noise-free. The resultant image is an edge detected binary image.

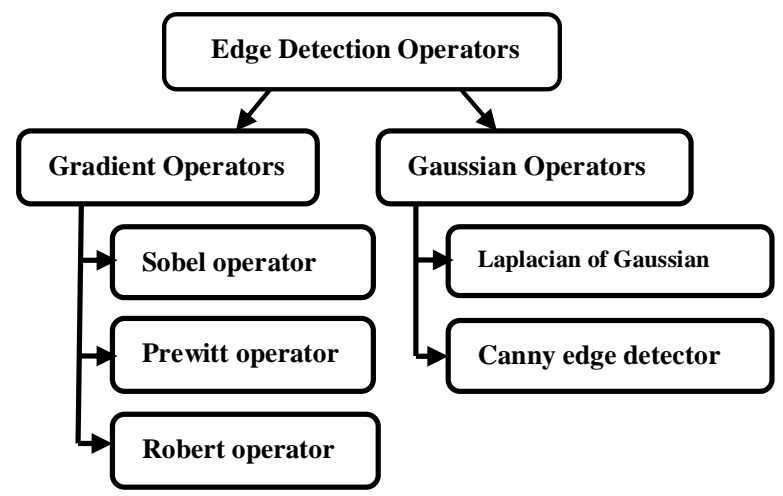

Fig : 2.1 Types of Edge Detection Operators

In a region-based [2] [4] [10] segmentation, an image features are associated with pixels are form a region. Region-based features are extracted and used to define "classes". The various techniques are: Local techniques, Global techniques and Splitting and Merging techniques. The main approaches are based on 1.Thresholding,
2.Region growing 3.Region splitting/ merging 4.Clustering in feature space. Region growing [11] [12] is making groups of pixels to form a sub-regions and larger regions.

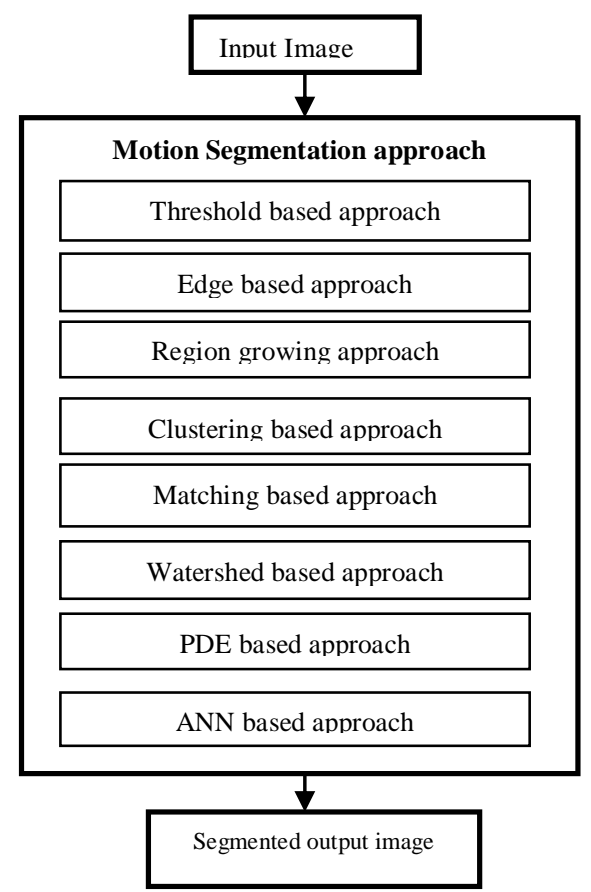

Fig 2.2 General Methodologies available for Segmentation

The regions are grow by adding seed points (it is a form of aggregated pixels) to adjacent pixels with relevant properties like gray level, texture, color, shape. Region growing techniques gives better results in noisy images when compare to edge detection methods.

Watershed Method [15] Segments the image, where regions-of-interest are closes to one another. Here, an image is treated as a landscape with ups and downs; it is represented by different gray values of the respective pixels.

\section{B. Related Work}

The [13] describes the various image segmentation techniques. These techniques are suitable for many medical image applications. These techniques can be used for object recognition and detection. An automatic threshold-based liver laceration segmentation method is proposed in [14]. Pre-contrast and post-contrast images are considered to evaluate the accuracy. The Lower threshold and higher threshold are calculated by statistical and texture measures. In post-contrast image, the gray level difference of liver and liver lesion is comparatively tough than the precontrast image where it is thin, it makes segmentation is difficult. The diverse size of lesions in pre-contrast and post-contrast images is segmented and also it improves the radiological analysis. An Improved Watershed Image 


\section{International Journal of Engineering Technology and Management Sciences[IJETMIS]}

Website: ijetms.in Issue:5, Volume No.4, September-2020 DOI: 10.46647/ijetms.2020.v04i05.012

Segmentation Technique [15] performs group of image elements with same characteristics, i.e. dividing an image into number of regions or objects with similar properties. Further interpretation processes like object recognition and classification are closely related with the quality of the segmentation process. Alternatively the landscape immersed in a lake, with holes penetrate in local minima. Dams are filled by water, coming from different catchment basins, until it reaches the highest level. The landscape is divided into regions of basins separated by dams, called watersheds. The problems in traditional watershed method will be solved by using pre-processing technique to minimize the noise and regulate the image intensity. Image Segmentation Techniques [16] says that this process of segmentation will assign a single value to each pixel of an image to make a difference between regions in an image. It can be done by the properties like color, intensity, and texture of an image. According to domain image segmentation techniques is selected. Image segmentation is plays an important role in all the field like medical images, satellite imaging, machine vision, biometrics, and military, image retrieval extracting features and recognizing objects from the image.

\section{SYSTEM IMPLEMENTATION AND EXPERIMENTAL RESULTS}

Threshold-based segmentation is the basic method of image segmentation used to create binary images. The segmentation threshold is converts the color image into binary image and it divides an image into segments with respect to intensity value with one color or grayscale value to define its boundary. Global Threshold can perform with a appropriate threshold value (T) and it is constant for the whole image. Based on $\mathrm{T}$ the output image $q(x, y)$ can be obtained from the original image $p(x, y)$. The threshold value in variable threshold method is change over in an image. They are Local Threshold, here $\mathrm{T}$ rely upon the neighborhood of $\mathrm{x}$ and $\mathrm{y}$. In adaptive Threshold method the value $\mathrm{T}$ is a function of $\mathrm{x}$ and $\mathrm{y}$ and multiple threshold values $\mathrm{T} 0$ \& $\mathrm{T} 1$ are used in multiple threshold method. Fig 3.1 depicts the general flow of the threshold segmentation.

TABLE I

SEQUENCE OF STEPS IN THRESHOLD SEGMENTATION

\begin{tabular}{|c|c|c|c|}
\hline Test steps & Expected result & Actual Result & $\begin{array}{c}\text { Status } \\
\text { (pass/ } \\
\text { fail) }\end{array}$ \\
\hline Image conversion & Gray image & Gray image & Pass \\
\hline Gray scale level & Level shown & Level $=0.00003$ & Pass \\
\hline Thresholding image & Output image & Output image & Pass \\
\hline
\end{tabular}

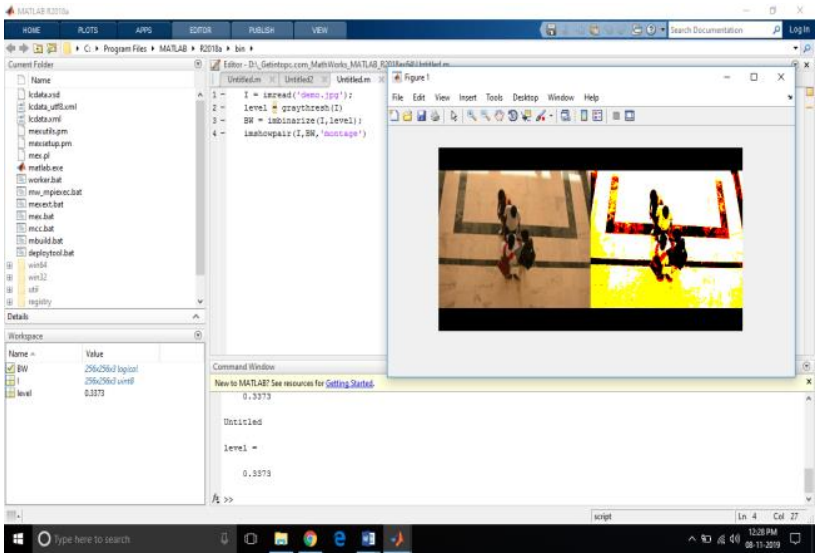

Fig 3.2a:Output of threshold segmentation on human being in a image

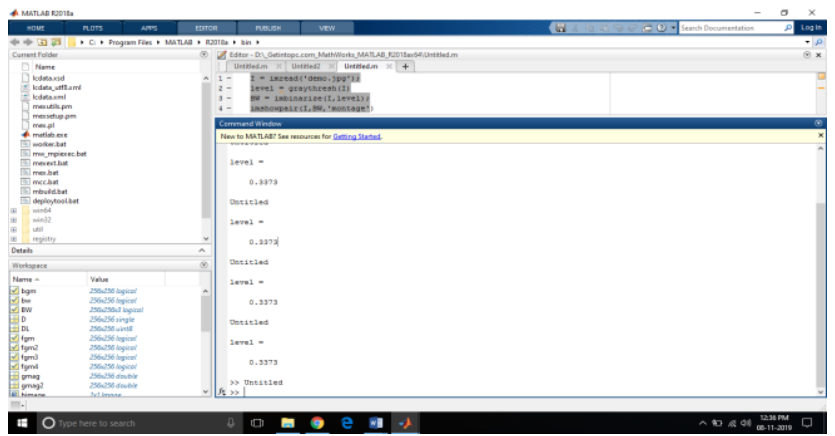

Fig 3.2b: Output of threshold segmentation on human being in an image

Edge-based Segmentation is used to separate the images using different types of gradient and Gaussian operators. Table II shows the advantages and disadvantages of edgebased operators.

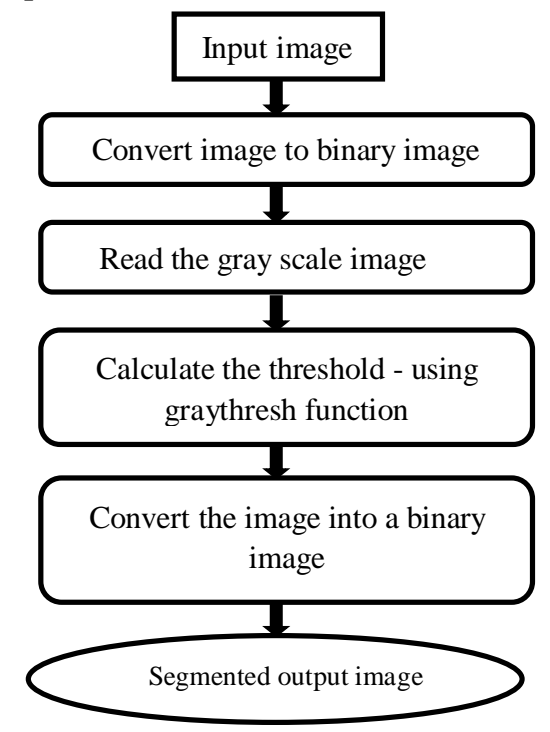

Fig 3.1. General Flow of Threshold Segmentation 


\section{International Journal of Engineering Technology and Management Sciences[IJETMSS]}

Website: ijetms.in Issue:5, Volume No.4, September-2020 DOI: 10.46647/ijetms.2020.v04i05.012

Table II: Advantages and Disadvantages of Edge based segmentation operators

\begin{tabular}{|c|c|c|c|}
\hline \multicolumn{2}{|c|}{$\begin{array}{c}\text { Name of the } \\
\text { Operator }\end{array}$} & Merits & Demerits \\
\hline \multirow[t]{6}{*}{ Gradient } & \multirow[t]{2}{*}{ Sobel } & \multirow{2}{*}{$\begin{array}{l}\text { Easy for } \\
\text { searching } \\
\text { smooth edges }\end{array}$} & Sensitive to noise \\
\hline & & & $\begin{array}{l}\text { Detect with thick and } \\
\text { rough edges does not } \\
\text { give appropriate } \\
\text { results }\end{array}$ \\
\hline & \multirow[t]{2}{*}{ Prewitt } & \multirow[t]{2}{*}{$\begin{array}{l}\text { Best operator } \\
\text { to detect the } \\
\text { orientation of } \\
\text { an image }\end{array}$} & $\begin{array}{l}\text { The magnitude of the } \\
\text { coefficient is fixed } \\
\text { and cannot be } \\
\text { changed. }\end{array}$ \\
\hline & & & $\begin{array}{l}\text { Diagonal direction } \\
\text { points are not } \\
\text { preserved. }\end{array}$ \\
\hline & \multirow[t]{2}{*}{ Robert } & \multirow{2}{*}{$\begin{array}{l}\text { Diagonal } \\
\text { direction } \\
\text { points are } \\
\text { preserved }\end{array}$} & $\begin{array}{l}\text { Very sensitive to } \\
\text { noise }\end{array}$ \\
\hline & & & $\begin{array}{l}\text { Not very accurate in } \\
\text { edge detection }\end{array}$ \\
\hline \multirow[t]{3}{*}{ Gaussian } & $\begin{array}{l}\text { Laplacian } \\
\text { of } \\
\text { Gaussian } \\
\text { (LoG) }\end{array}$ & $\begin{array}{l}\text { Preserve the } \\
\text { orientation }\end{array}$ & $\begin{array}{l}\text { Very sensitive to } \\
\text { noise }\end{array}$ \\
\hline & \multirow[t]{2}{*}{ Canny } & $\begin{array}{l}\text { Less } \\
\text { Sensitive to } \\
\text { noise }\end{array}$ & \multirow[t]{2}{*}{$\begin{array}{l}\text { Complex computation } \\
\text { and time consuming }\end{array}$} \\
\hline & & $\begin{array}{l}\text { It extracts } \\
\text { image } \\
\text { features } \\
\text { without } \\
\text { altering the } \\
\text { features }\end{array}$ & \\
\hline
\end{tabular}

The results of edge segmentation method is shown Fig $3.3 \mathrm{a}, \mathrm{b}, \mathrm{c}$. In an input image, there is 2 persons in it and detected by the different operators.

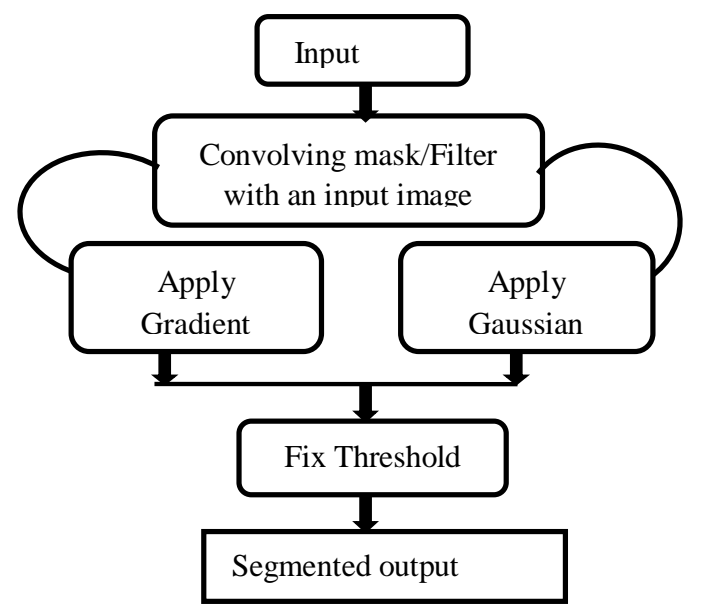

Fig 3.3. General Flow of Edge based Segmentation

In Watershed Segmentation Method, an image is treated as a topographic landscape with hill and slope.The elevation values of the landscape are typically defined by the gray values of the respective pixels or their gradient magnitude.

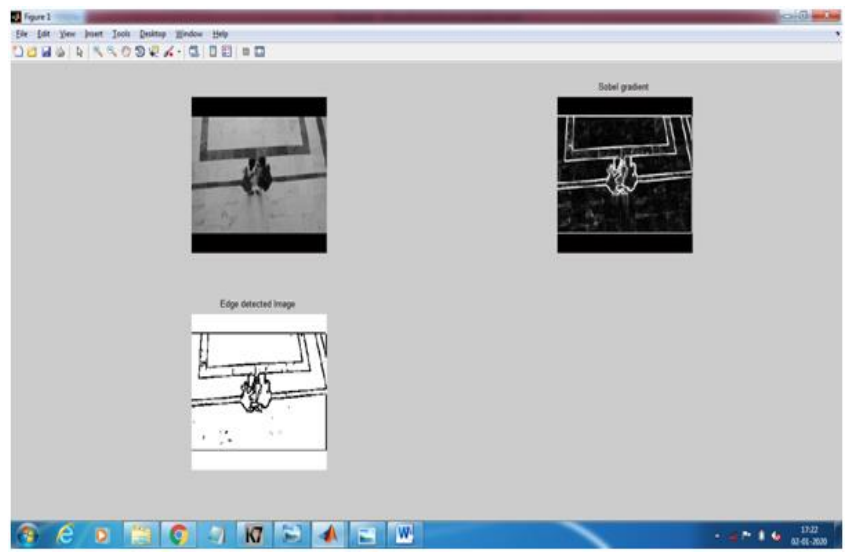

Fig :3.3a. Output of Sobel operator

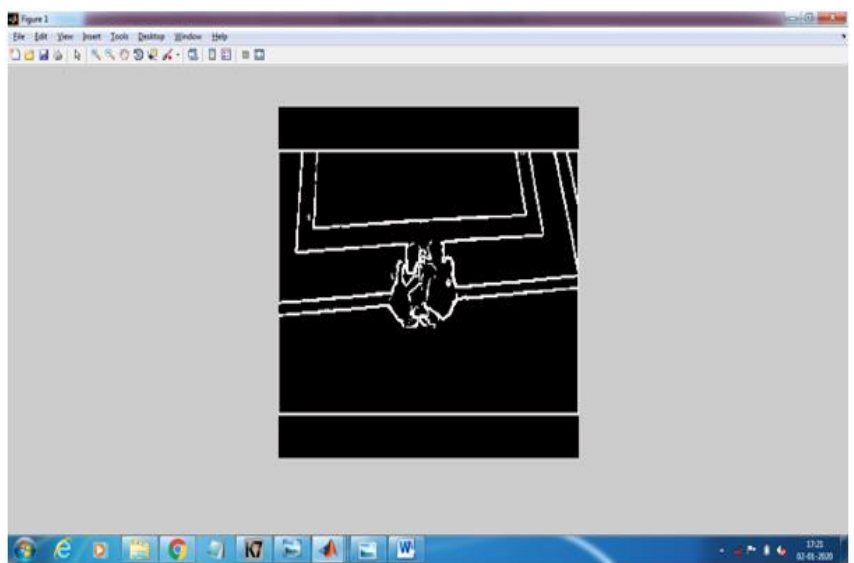

Fig : 3.3b. Output of prewitt operator

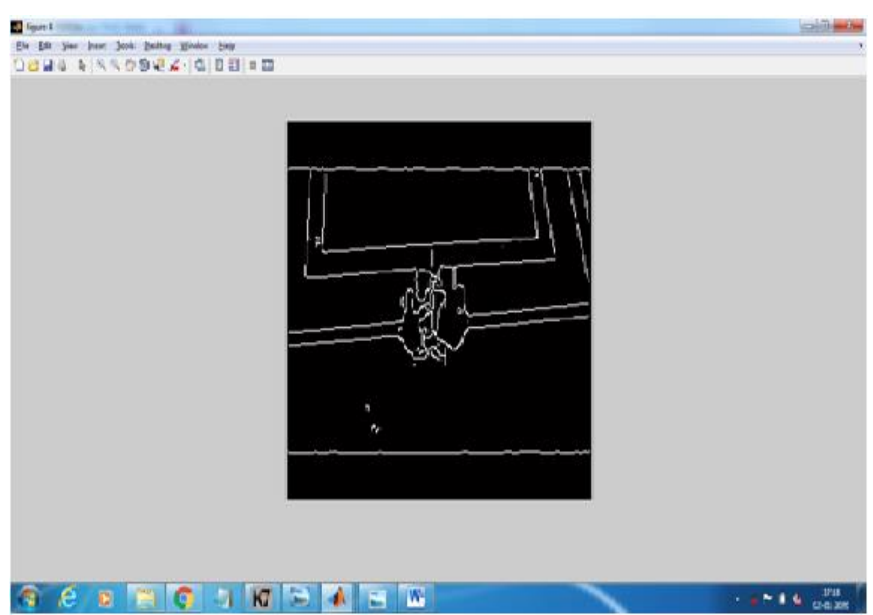

Fig :3.3c. Output of canny operator 


\section{International Journal of Engineering Technology and Management Sciences[IJETMSS]}

Website: ijetms.in Issue:5, Volume No.4, September-2020 DOI: 10.46647/ijetms.2020.v04i05.012

Table III : Sequence of steps in watershed Segmentation

\begin{tabular}{|l|l|l|c|}
\hline Test steps & Expected result & Actual Result & $\begin{array}{c}\text { Status } \\
\text { (pass/fail) }\end{array}$ \\
\hline $\begin{array}{l}\text { Image } \\
\text { conversion }\end{array}$ & Gary image & Gray image & Pass \\
\hline $\begin{array}{l}\text { Gradient } \\
\text { magnitude }\end{array}$ & Image shown & Image shown & Pass \\
\hline $\begin{array}{l}\text { Watershed } \\
\text { transform of } \\
\text { gradient } \\
\text { magnitude }\end{array}$ & Image shown & Image shown & pass \\
\hline
\end{tabular}

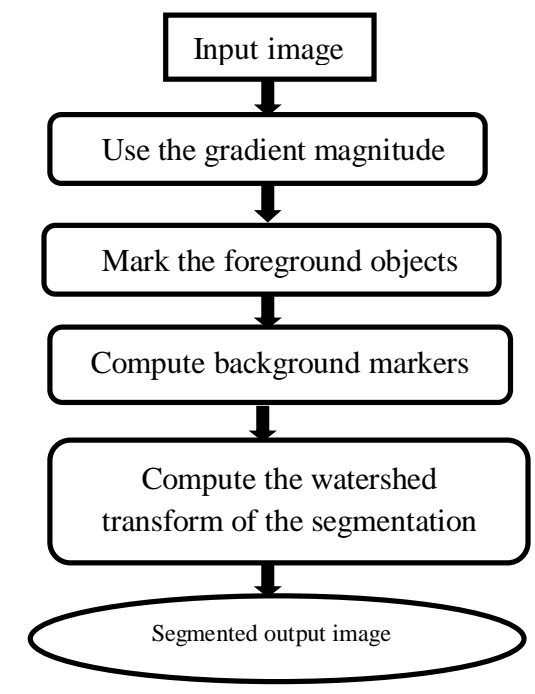

Fig 3.4. General Flow of watershed Segmentation

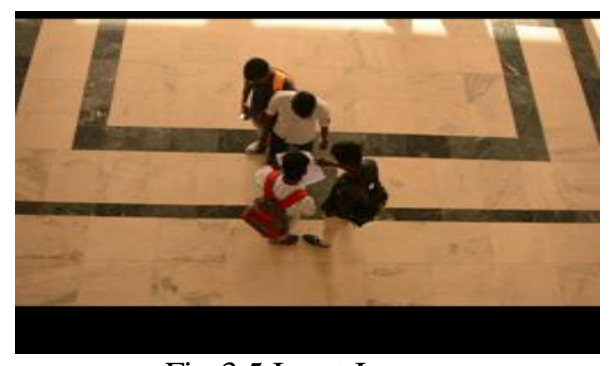

Fig:3.5 Input Image

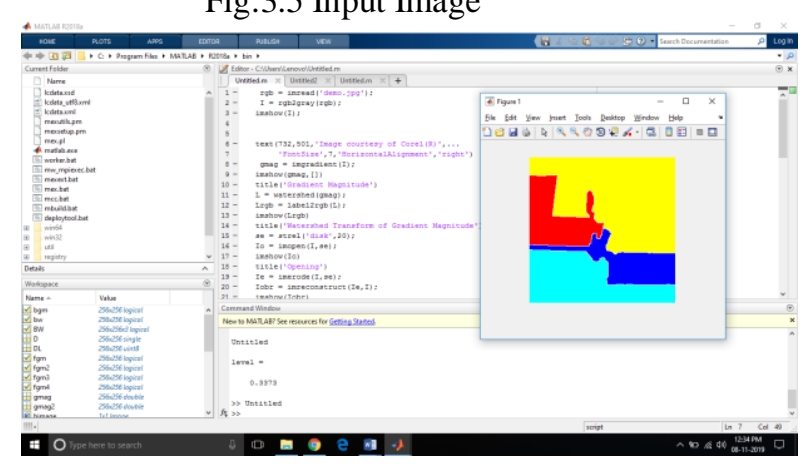

Fig: 3.5a. Output of watershed segmentation

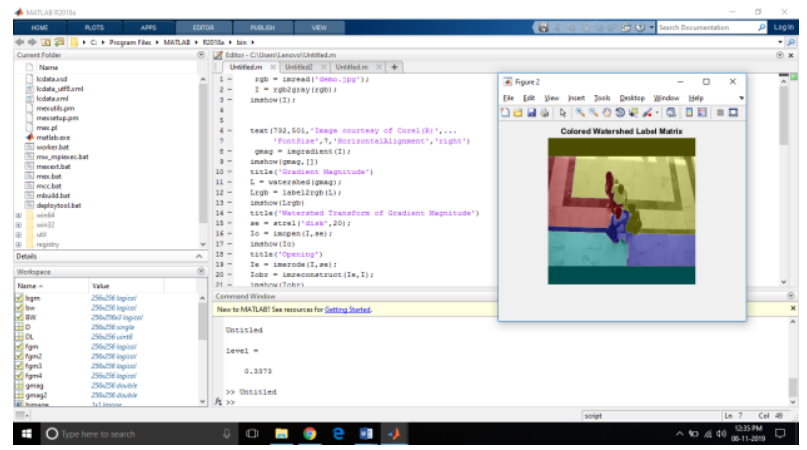

Fig: 3.5b.Output of colored watershed segmentation

\section{CONCLUSION}

In this study, various image segmentation techniques are described and compared. These techniques are suitable for object recognition, detection. and medical image analysis applications. In medical images, it can be used to detect cancer and in satellite images detects roads and bridges. Thus, methods are suitable for various types of image applications. Image segmentation is popularly worked in many applications and it has a demanding future.

\section{References}

[1] Chang-TsunLi, Randy Chiao, Multi-resolution Genetic Clustering Algorithm for Texture Segmentation Image and Vision Computing 2003, 21:955-9660.

[2] R. C. Gonzales, R. E. Woods, and S. L. Eddins, Digital Image Processing using MATLAB, Pearson Education, 2008.

[3] S. Aksoy, "Image Segmentation", Department of Computer Engineering, Bilkent University .

[4] K. G. Gunturk , "EE 7730 - Image Analysis I", Louisiana State University.

[5] F. Z. Kettaf, D. Bi, and J. P. Asselin de Beauville, "A comparison study of image segmentation by clustering technique s", IEEE Trans, vol. 2,1996, pp. 1280-1283.

[6] M. Xiao, L. Zhang, A background reconstruction algorithm based on modified basic sequential clustering, in: International Colloquium on Computing, Communication, Control, and Management, CCCM 2008, vol. 1, August 2008, pp. 47-51.

[7] Deekshitha P, B Tarun Reddy, ShravaniBadadha, K KDhruthi, S PandiarajObject Motion Perception and Tracking Using Sift With KMeans Clustering Journal of Network Communications and Emerging Technologies (JNCET) www.jncet.org Volume 8, Issue 4, April (2018).

[8] L. Aurdal, "Image segmentation beyond thresholding", Norsk Regnesentral , 2006.

[9] Y. Zhang, H. Qu, and Y. Wang, "Adaptive Image Segmentation Based on Fast Thresholding and Image Merging", Artificial Reality and Telexistence--Workshops, pp. 308-311.

[10] Y. Chang, and X. Li, "Adaptive Image Region-Growing," IEEE Trans . on Image Processing, vol. 3, no. 6,1994.

[11] T. Gevers, and V. K. Kajcovski, "Image segmentation by directed region subdivision", Proceedings of the 12th 1APR International Conference on Pattern Recognition, vol. 1, 1994. 
Website: ijetms.in Issue:5, Volume No.4, September-2020 DOI: 10.46647/ijetms.2020.v04i05.012

[12] Dilpreet Kaur ,and Yadwinder Kaur," Various Image Segmentation Techniques: A Review" IJCSMC, Vol. 3, pg.809 - 814.

[13] Asmita A. Moghe, Jyoti Singhai \& S.C Shrivastava," Automatic Threshold based Liver Lesion Segmentation in Abdominal 2D-CT Images Processing" (IJIP), Volume (5).

[14] Anju Bala," An Improved Watershed Image Segmentation Technique using MATLAB" Volume 3, Issue 6, ISSN 2229-5518".

[15] Waseem Khan," Image Segmentation Techniques: A Survey" Journal of Image and Graphics Vol. 1.

[16] Muhammad Waseem Khan," A Survey: Image Segmentation Techniques" Vol. 3, No. 2, DOI: 10.7763/IJFCC.2014.V3.274. 